\title{
Short Interpregnancy Interval and Low Birth Weight
}

\author{
SUMAIRA IQBAL ${ }^{1}$, SHAMAILA SHAMAUN ${ }^{2}$, AFSHAN SHAHID ${ }^{3}$, RIFFAT JALEEL $^{4}$, YASMEEN GULL $^{5}$, MEHREEN IQBAL ${ }^{6}$ \\ ${ }^{1}$ Senior Registrar, Department of Obstetrics and Gynaecology, Lady Dufferin Hospital, Karachi \\ ${ }^{2,3}$ Assistant Professor, Department of Obstetrics and Gynaecology Unit 2 DIMC/DUHS, Karachi \\ ${ }^{4}$ Professor and Head of Department of Obstetrics and Gynaecology Unit 3 DMC/Dr Ruth KM Pfau Civil Hospital, Karachi \\ ${ }^{5}$ Assistant Professor Department of Obstetrics and Gynaecology, Meer Jam Ghulam Qadir Hospital, Hub District Lasbella Balochistan \\ ${ }^{6}$ Senior Registrar Department of Obstetrics and Gynaecology Unit 2, DIMC/DUHS, Karachi \\ Corresponding author: Shamaila Shamaun, Email: shamaila.shamaun@duhs.edu.pk, Cell: 03002123690
}

\begin{abstract}
Objective: To determine the frequency of Low Birth Weight among women with short interpregnancy interval.

Study Design: Descriptive case series.

Study Setting: Study was conducted at Department of Gynecology and Obstetrics Unit 1, Civil Hospital, Karachi.

Duration of Study: Six months after approval of synopsis from 22-12-15 till 22-06-16.

Subjects and Methods: Data was prospectively collected from patients after taking a verbal consent. 384 women with short interval pregnancy were included. Quantitative data was presented as simple descriptive statistics giving mean and standard deviation and qualitative variables was presented as frequency and percentages. Effect modifiers were controlled through stratification to see the effect of these on the outcome variable. Post stratification chi square test was applied taking $p$-value of $\leq 0.05$ as significant.

Results: Out of 384 patients mean age and BMI in our study was $29.56 \pm 3.91$ years and $30.72 \pm 1.34 \mathrm{~kg} / \mathrm{m} 2$ respectively. 135 (35.2\%) and 249 (64.8\%) had and did not have low birth weight respectively.

Conclusion: Our study confirmed that short IPI is an independent risk factors for adverse pregnancy outcomes like low birth weight. These finding emphasize the importance of providing support for family planning programs which will support optimal IPI and improve pregnancy outcomes.
\end{abstract}

Keywords: Short interpregnancy interval and low birth weight.

\section{INTRODUCTION}

Maternal and foetal risks are increased when the time between pregnancies is too short [1]. Many family planning professionals have long recognised that a two-year birth interval is critical for the health of the mother, child, and newborn [2]. Short interpregnancy intervals ( 0 to 5 months) have been found to increase the risk of preterm delivery and low birth weight in the second pregnancy [1].

Short interpregnancy intervals were found to increase the risk of infant death in a recent study from India and Pakistan [3-4]. Between 18 and 24 months between pregnancies, the lowest maternal and foetal risks have been suggested [3]. A study in Ohio-USA found that, regardless of gestational age at birth, interpregnancy gap is a major contributor to newborn morbidity [34].

As a major epidemiological issue, low birth weight (the percentage of infants weighing less than 2500 grammes at delivery) is critical since it increases the risk of several diseases in adulthood. Birth spacing is also a contributing factor to undesirable birth outcomes. According to a Polish study, mothers with short interpregnancy intervals are more likely to give birth to underweight children [6].

When it comes to infant and maternal health outcomes, short birth intervals have long been seen as a risk factor. New research on optimal birth spacing collected and commissioned by the CATALYST Consortium has corroborated this long-held belief [2]. Birth intervals of less than five months were shown to be linked with low birth weight in a study conducted at Sheikh Zaid Hospital, Larkana [7]. Low-birth weight was found to be 48 percent more common in women who gave birth within a 5-month period [7].

Pakistan has the second highest recorded rate of low birth weight in the world (32 percent) according to the UNICEF 2013 State of the World's Children Report. Pakistani women have a birth gap of less than two years for about a third of them [8].

It has been shown that low birth weight has a negative impact on a child's health and well-being in the womb, as well as later in life.

In earlier studies, it has been found that a short interpregnancy interval has a synergistic effect on the likelihood of having a low birth weight baby. Up to this point, very few nationwide studies have been carried out. As a result, the goal of this study is to investigate the frequency of low birth weight in women with short interpregnancy intervals. In this study, we hope to encourage women to space their pregnancies out, educate them about the risks of a short delivery gap, and promote the best possible outcomes for both mother and child. Reduce the incidence of preterm and low birth weight children, improve mother health, and promote healthy childhood development as a result of this.

\section{MATERIALS AND METHODS}

This descriptive case series study was conducted at Department of Gynecology and Obstetrics Unit 1, Civil Hospital, Karachi, duration was six months from 22-12-15 till 22-06-16. A total of 384 short interval pregnant individuals with singleton pregnancy were enrolled. Those that were admitted to the hospital were between the ages of 20 and 40 years. Following the receipt of signed informed consent, detailed demographic information, including age, gestational age, weight, parity, BMI, and a complete blood picture, was collected. Patients with intrauterine death (IUGR), multiple pregnancies (including multiple C-sections), emergency Csections (including polyhydramniosis), meconium-stained liquor (including meconium-stained liquor), Eclampsia (including Eclampsia), and premature rupture of membranes were excluded from the study.

Following the receipt of informed consent, only those patients were chosen, and basic investigations as well as ultrasound scan reports were evaluated, with only those patients meeting the inclusion criteria being included in the investigation. In less than an hour after delivery, qualified labour room staff weighed their newborns without clothing on an infant weighing scale in grammes; the scale was checked and zeroed before each weigh-in was performed. The information collected included the mother's age, parity, weight, and height, as well as the age of the last child born, the date of her last menstrual period, the projected date of delivery, and the gestational age.

SPSS Version 22 was used to analyse the data. The mean and standard deviations for maternal age, gestational age, mother height, weight, body mass index (BMI), and newborn weight were calculated. The percentages and frequencies for neonatal gender, parity, and low birth weight (yes/no) were estimated based on the data.

\section{RESULTS}

Out of 384 patients minimum age of the patient was 24 while maximum age of the patients was 35 years. Mean age in our study 
was 29.56 years with the standard deviation of \pm 3.91 . Whereas, mean gestational age, duration of short interval pregnancy, BMI, height and weight in our study was $37.72 \pm 3.39$ weeks, $4.21 \pm 1.01$ months, BMI $30.72 \pm 1.34 \mathrm{~kg} / \mathrm{m} 2,148 \pm 7.28 \mathrm{~cm}$ and $78.7 \mathrm{~kg}$ respectively. As shown in Table 1.

Table-1: Descriptive Statistics $\mathrm{n}=384$

\begin{tabular}{|l|l|l|l|}
\hline Variable & Mean & $\begin{array}{l}\text { Standard } \\
\text { Deviation }\end{array}$ & Min-Max \\
\hline Maternal Age (Years) & 29.56 & \pm 3.91 & $24-35$ \\
\hline Gestational Age (Weeks) & 37.72 & \pm 3.39 & $36-40$ \\
\hline $\begin{array}{l}\text { Duration of Short Interval } \\
\text { Pregnancy }\end{array}$ & 4.21 & \pm 1.01 & $1-5$ \\
\hline Body Mass Index (KG/M $\left.{ }^{2}\right)$ & 30.72 & \pm 1.34 & $27-33$ \\
\hline Height (cm) & 148 & \pm 7.28 & $148-162$ \\
\hline Weight (KG) & 78.7 & \pm 9.87 & $68-115$ \\
\hline
\end{tabular}

Frequency distribution of duration of short interval pregnancy showed that out of 384 short interval pregnancies, 91 (23.7\%) and $293(76.3 \%)$ were in the duration of short interval pregnancy group $<3$ months and $>3$ months respectively. As presented in Figure 2.

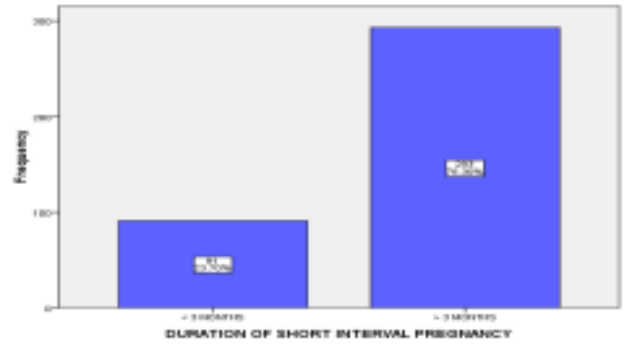

Figure-4: Duration of Short Interval Pregnancy Distribution $n=384$

Frequency distribution of low Birth weight showed that out of 384 short interval pregnancies, $135(35.2 \%)$ and $249(64.8 \%)$ had and did not have low birth weight respectively. As presented in Figure 3.

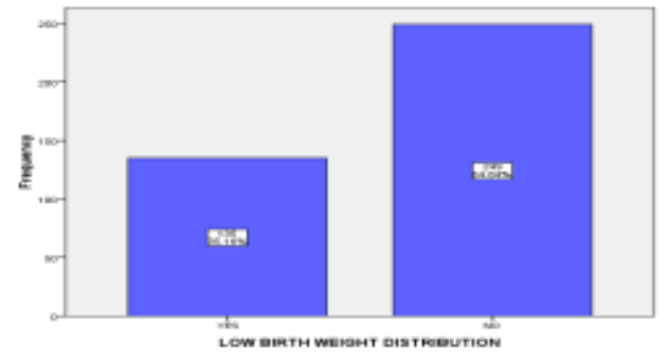

Figure-1: Low Birth Weight Distribution $n=384$

Stratification for duration of short interval pregnancy with respect to low birth weight showed that $26(19.3 \%)$ and $65(26.1 \%)$ in the patients with short interval pregnancy for $<3$ months had and did not have low birth weight respectively. Whereas 109 $(80.7 \%)$ and $184(73.9 \%)$ in the patients with short interval pregnancy for $>3$ months had and did not have low birth weight respectively. P-value was 0.08 . As presented in Table 2 .

Table-4: Duration of Short Interval Pregnancy According to Gestational Age $\mathrm{n}=384$

\begin{tabular}{|l|l|l|l|}
\hline $\begin{array}{l}\text { Duration of Short } \\
\text { Interval Pregnancy }\end{array}$ & Low Birth Weight & \multirow{2}{*}{ Total } \\
\cline { 2 - 3 } & Yes & No & $\begin{array}{l}91 \\
(23.7 \%)\end{array}$ \\
\hline$<3$ Months & $26(19.3 \%)$ & $65(26.1 \%)$ & $\begin{array}{l}293 \\
(76.3 \%)\end{array}$ \\
\hline$>3$ Months & $109(80.7 \%)$ & $184(73.9 \%)$ & $\begin{array}{l}384 \\
(100 \%)\end{array}$ \\
\hline Total & $135(100 \%)$ & $249(100 \%)$ & \multicolumn{3}{|l}{} \\
\hline P-Value & 0.08 & \multicolumn{3}{|l}{} \\
\hline
\end{tabular}

\section{DISCUSSION}

The inter-pregnancy interval (IPI) is defined as the period of time that elapses between two successive pregnancy. Pregnancy that is not scheduled properly increases the health risks for both the mother and the child, whereas the optimal interpregnancy interval (IPI) is a critical driver of maternal health and pregnancy outcomes in women. However, it should be noted that the categorization of IPI varies slightly between investigations, making comparisons between data from different studies difficult [11-12].

Out of 384 patients in our study, the mean age and BMI were 29.563 .91 years and $30.721 .34 \mathrm{~kg} / \mathrm{m} 2$ respectively, according to the results of our research. Low birth weight was seen in 135 (35.2 percent) and 249 (64.8 percent) of the participants, respectively.

According to the findings of the Basso et al study, short interpregnancy intervals (less than or equal to 8 months) were related with preterm birth but not with low birth weight. When comparing deliveries between 24 and 36 months, the adjusted odds ratios for preterm birth were 3.60 (95 percent confidence interval 2.04 to 6.35 ) for intervals up to 4.00 months and 2.28 (1.49 to 3.48 ) for intervals between 4.01 and 8.00 months. When comparing deliveries between 24 and 36 months, the risk of preterm birth was 3.5 percent. Women who had previously had a full-term pregnancy were at greater risk. Social class, age, and parity were all taken into consideration [9].

The researchers conducted another study that included 2,253 pregnancies and found that one-third (35 percent) of them were conceived within 18 months of a previous birth. When sociodemographic and childbearing characteristics were taken into account, women who were aged 15-19 years or married at the time of conception of the index pregnancy, who began childbearing after the age of 30 , and who reported the pregnancy as unintended were significantly more likely to have a short interpregnancy interval than those who were not. Women from higher socioeconomic classes were more prone than others to plan short interpregnancy intervals (married, non-Hispanic white, college educated, or non-Medicaid delivery). If we could avoid unwanted pregnancies, we believe we could lower the number of women who have short interpregnancy intervals from 35 percent to 23 percent [10].

According to the findings of the Bener et al study, moms with a short interpregnancy gap ( 40.3 percent) had a higher chance of having low birth weight babies, whereas mothers with a longer interpregnancy interval of 24 months had a lower risk of having normal birth weight babies (44.7 percent). An interpregnancy interval of 612 months was more common among women under the age of $25(49.4 \% ; \mathrm{p} 0.001)$ and those who were illiterate $(13.1 \%)$ than among the controls, and these women had a higher chance of having a baby of low birth weight when compared to the controls. Women who gave birth to low birth weight children received less prenatal care throughout the first trimester (p0.001). When comparing cases (58.7 percent) and controls (79 percent), it was shown that women with a short birth interval were less likely to have a normal delivery $(p=0.001)$. Low birth weight was found to be associated with a J-shaped relationship with the interpregnancy interval in one study [13].

According to another Tanzanian study, the median IPI was 36 months. Short interpregnancy intervals (24 months) were found to be associated with preterm delivery (OR 1 - 52; 95 percent confidence interval [Cl] 1.31-1.74), low birth weight (OR 1 • 61; 95 percent confidence interval $[\mathrm{Cl}] 1 \cdot 34-1.72$ ), and perinatal death (OR 1 - 63; 95 percent confidence interval [Cl] 1.22-1.91) when compared with IPIs of 24-36 months (reference group). A longer IPI of 37-59 months or more was similarly associated with increased chances of preterm birth and low birth weight, but not with increased risks of perinatal mortality [14].

It is important to note that both short and long IPI are independent risk factors for poor pregnancy outcomes. These findings highlight the necessity of giving assistance for family 
planning programmes that will help women achieve optimal IPI and have better pregnancies and birth outcomes.

\section{CONCLUSIONS}

Both short and long IPIs are associated with an increased risk of unfavourable pregnancy outcomes, such as underweight babies, according to our research. Pregnancy outcomes and foetal survival can be improved with the help of this research. Women who have recently given birth should be identified and counselled by health care providers about the impact of IPIs (short or long) and the risk of subsequent pregnancy to optimise the interval for favourable pregnancy outcomes. These findings underscore the need of supporting family planning services in order to achieve optimal IPI and improve pregnancy outcomes.

\section{REFERENCES}

1. de Weger FJ, Hukkelhoven CWPM, Serroyrn J, teVelde ER, Smits LJM. Advanced maternal age, short interpregnancy interval, and perinatal outcome. Am J ObstetGynecol 2011;204:421.e1-9

2. Available h_Spacing_Optimal_Interval

3. DeFranco EA, Seske LM, Greenberg JM, Muglia LJ. Influence of interpregnancy interval on neonatal morbidity. Am J ObstetGynecol 2015;212:386.e1-9

4. Salihu HM, August EM, MbahAK,deCuba RJ 2nd, AlioAP,RowlandMishkit V, et al. The impact of birth-spacing on subsequent feto-infant outcomes among community enrollees of a federal healthy start project. J Community Health 2012;37:137-42
5. Merklinger-Gruchala A, Jasienska G, Kapiszewska M.(2015). Short interpregnancy interval and low birth weight: a role of parity. Am J Hum Biol., 2015. 27:660-666 doi : 10.1002/ajhb.22708

6. UN Children's Fund (UNICEF) 2013 State of the World's Children Report doi : southasia.oneworld.net>Resources

7. Bhatti A, Naz S, Majid E, Bhatti N. Maternal risk factors associated with low birth weight babies. Med Channel Apr-Jun 2010;16(2):334-8

8. Kamal A, Pervaiz MK.(2012) Determinants of higher order birth intervals in Pakistan. ISSN 1684-8403 Journal of Statistics Volume 19, 2012 pp 54-82.

9. Yaremco E, Inglis A, Innis SM, Hippman C, Carrion P, Lamers Y, et al. Red blood cell folate levels in pregnant women with a history of mood disorders: A case series. Birth Defects Res A: ClinMolTeratol. 2013;97(6):416-420.

10. Zhu BP. Effect of interpregnancy interval on birth outcomes: Findings from three recent US studies. Int J Gynecol Obstet. 2005;89:S25S33.

11. Basso O, Olsen J, Knudsen LB, Christensen K. Low birth weight and preterm birth after short interpregnancy intervals. Am J Obstet Gynecol. 1998 Feb;178(2):259-63.

12. Gemmill A, Lindberg LD. Short interpregnancy intervals in the United States. Obstet Gynecol. 2013 Jul;122(1):64-71.

13. Bener A, Saleh NM, Salameh K, Mohd K, Basha B, Joseph S et al The impact of the interpregnancy interval on birth weight and other pregnancy outcomes. Rev. Bras. Saude Mater. Infant.2012 Sep;12(3):233-41.

14. Mahande MJ, Obure J. Effect of interpregnancy interval on adverse pregnancy outcomes in northern Tanzania: a registry-based retrospective cohort study. BMC Pregnancy Childbirth.2016; 16: 140. 